\title{
Clonal Expansion of Bone Marrow CD8+ T Cells in Acute Myeloid Leukemia Patients at New Diagnosis and Post Chemotherapy
}

\section{Zhongxin Feng}

Guizhou Medical University

\section{Qin Fang}

Guizhou Medical University

\section{Xingyi Kuang}

Guizhou Medical University

Xin Liu

Guizhou medicine university

Ying Chen

Guizhou medicine University

\section{Dan Ma}

Guizhou medicine University

Jishi Wang ( $\nabla$ wangjishi9646@163.com )

Guizhou Medical University

\section{Research}

Keywords: CD8+ T cell, T cell receptor, bone marrow, programmed death-1, acute myeloid leukemia

Posted Date: August 2nd, 2020

DOl: https://doi.org/10.21203/rs.3.rs-50231/v1

License: (9) This work is licensed under a Creative Commons Attribution 4.0 International License. Read Full License 


\section{Abstract}

Background: $\mathrm{CD} 8^{+} \mathrm{T}$ cells are crucial adaptive immune effectors and express receptors (T cell receptors, TCRs) that specifically recognize and eradicate tumor cells. The diversity of the TCR repertoire is generated by specialized genetic diversification mechanisms, leading to an extremely variable TCR repertoire capable of recognizing a wide range of antigens. However, the variations in $\mathrm{CD} 8^{+} \mathrm{TCR}$ diversity and their clinical implications in AML patients remain unknown.

Methods: $\mathrm{CD}^{+} \mathrm{T}$ cells in 10 healthy donors and $31 \mathrm{AML}$ patients at diagnosis and after chemotherapy were enriched using the Dynabeads CD8 Positive Isolation Kit. Flow cytometry were used for PD-1 expression level analysis of $\mathrm{CD} 8^{+} \mathrm{T}$ cells and CD $8^{+} \mathrm{PD}-1^{+}$and $\mathrm{CD} 8^{+} \mathrm{PD}-1^{-} \mathrm{T}$ cell sorting. TCR $\beta$ deep sequencing was performed to analysis the $\mathrm{CD} 8^{+} \mathrm{T}$ cells clonal expansion and TCR repertoire diversity.

Results: Diminished TCR repertoire diversity and expansional T cell clones were noted in the bone marrow of AML patients. In relapsed patients, $T$ cells were found to be more clonally expanded post chemotherapy when compared to new diagnosis. Moreover, more significantly expanded TCR $\beta$ clonotypes were noted in $\mathrm{CD} 8^{+} \mathrm{PD}-1^{+} \mathrm{T}$ cells than in $\mathrm{CD} 8^{+} \mathrm{PD}-1^{-} \mathrm{T}$ cells regardless of the time point of examination.

Conclusions: Our systematic T-cell repertoire analysis may help better characterize $\mathrm{CD} 8^{+} \mathrm{T}$ cells pre- and post-chemotherapy in $\mathrm{AML}$, which may provide insights into therapeutic strategies in hematological malignancies.

\section{Background}

Acute myeloid leukemia (AML) is a type of progressive malignant disorder that is often associated with poorer survival rates. During the last several decades, little has changed in induction chemotherapy used to treat AML. While the majority of AML patients achieve a complete response (CR) at the outset, most of them fail to sustain such responses for a long time due to chemoresistance [1]. Considerable efforts have been dedicated to elucidating the drug resistance mechanisms in leukemia, but little attention has been paid to the discrepancies of chemotherapy in regulating the signals of immune system between responsive patients and non-responsive patients [2]. Cancer immunotherapy as a novel therapeutic approach has attracted considerable attention because of mounting successes in checkpoint blockade intervention and T cell engineering [3]. Potential new immunotherapeutic modalities hold much promise in the treatment of AML and are likely to increase in the future [4-8]; on the other hand, many significant challenges remain $[9,10]$. Hence, characterization of the immune and biological features of the disease is the key to optimizing the therapeutic strategy for AML patients.

$\mathrm{CD}^{+} \mathrm{T}$ cells are the main cytotoxic effector cells that mediate apoptosis through the $\mathrm{T}$ cell receptor (TCR), recognizing antigenic peptides bound to MHC class I molecules, which are expressed by tumor cells [11]. The TCR is made of two constituent chains ( $a \beta$ or $\gamma \delta)$, which undergo a $V(D) J$ recombination 
process. As the most diverse portion of the variable region, complementarity determining region 3 (CDR3) primarily contributes to the specificity of antigen recognition of $T$ cells $[12,13]$. Recent studies suggest that $\delta-C D R 3 s$ in $\gamma \delta T$ cells can be predictive of clinical responses in AML patients $[14,15]$.

Notwithstanding the potential as a therapeutic target, $C D 8^{+}$TCR repertoire dynamics and the modulation mechanisms of chemotherapy are still poorly understood. a $\mathrm{T}$ cells are the predominant subset, making up approximately $90-95 \%$ of all peripheral T cells in healthy adults [16]. Therefore, it is crucial to assess the characteristics of the $\mathrm{CD} 8^{+} \mathrm{TCR}$ repertoire and investigate the clinical significance in AML patients.

It has become increasingly clear that programmed death-1 (PD-1) is a key regulator of T cell dysfunction that arises from exposure to antigen stimulation [17]. Recent study has reported functional skewing of $\mathrm{CD}^{+} \mathrm{T}$ cells in newly diagnosed AML patients, which is correlated with an upregulation of PD-1 expression [18]. As expected, the response to chemotherapy is associated with upregulation of costimulatory molecules and attenuation of apoptotic signaling [19]. Compared to the new diagnosis, a significantly higher PD-1 expression level on $C D 8^{+} T$ cells is noted at the time of relapse. However, treatment with PD-1 pathway blockade or costimulatory molecule OX40 result in invigoration of CD8 ${ }^{+} \mathrm{T}$ cell effector function $[2,6,20]$. These data are consistent with previous findings that PD- $1^{+} \mathrm{CD} 8^{+} \mathrm{T}$ cells are implicated in AML progression and may be harnessed for reinvigorating cytotoxicity.

The diversity of the TCR repertoire may reflect the differential recognition of neoantigens. The PD- $1^{+}$CD $8^{+}$ phenotype has been identified as a marker of $T$ cell exhaustion and has been proven to display neoantigen-specific cytotoxic activity [21,22]. A diversified TCR repertoire enables the host immune system to recognize a large variety of tumor neoantigens [23]. Hence, an improved understanding of the $\mathrm{PD}-1^{+} \mathrm{CD} 8^{+} \mathrm{TCR}$ repertoire would enable more effective clinical and therapeutic strategies for AML patients.

In this study, we performed RNA sequencing of the CDR3 region from TCR $\beta$ chains of $\alpha \beta C D 8^{+} T$ cells and examined the different clonal expansion patterns between $\mathrm{AML}$ and healthy donor samples. In addition, we analyzed $\mathrm{T}$ cell clonality and distribution in paired patient samples collected at diagnosis and after chemotherapy. We further studied the TCR repertoire dynamics of PD-1-negative and PD-1-positive CD8 ${ }^{+}$ T cells. We found that TCR repertoire diversity was correlated with clinical outcomes. Collectively, these results underscored the antitumor reactivity of $C D 8^{+} T$ cells and the value of $C D 8^{+} T$ cell repertoire as a potential prognostic marker in AML, highlighting the importance of immune intervention.

\section{Materials And Methods}

\section{Samples}

The study was approved by the Institutional Review Board (IRB) of the Affiliated Hospital of Guizhou Medical University. Informed consent was obtained from all the subjects enrolled in the study. The clinical and demographic data for all the subjects are summarized in Supplementary Table 1. Peripheral blood 
mononuclear cells (PBMCs) and bone marrow mononuclear cells (BMMCs) were obtained from healthy donors and AML patients at diagnosis and post chemotherapy. Less than $5 \%$ blasts in BM by morphologic evaluation with neutrophil count greater than $1 \times 10^{9} / \mathrm{L}$ and platelet count greater than $1 \times$ $10^{11} / \mathrm{L}$ was considered complete remission (CR).

\section{Flow cytometry}

PBMCs or BMMCs were collected using Ficoll-Paque reagents (GE Healthcare, Sweden) via density gradient centrifugation, cryopreserved in $90 \%$ human serum, and formulated with $10 \%$ DMSO. CD $8^{+} \mathrm{T}$ cells were purified using the Dynabeads CD8 Positive Isolation Kit (Life Technologies, Norway) according to the manufacturer's protocol. The purity of sorted T cell samples was $>90 \%$ as determined by flow cytometry. PD- 1 expression level analysis of $C D 8^{+} T$ cells and $C D 8^{+} P D-1^{+}$and $C D 8^{+} P D-1^{-} T$ cell sorting was performed on BD Biosciences FACS Aria II.

\section{RNA isolation and TCR $\beta$ sequencing}

$\mathrm{CD}^{+} \mathrm{T}$ cells were enriched using the Dynabeads CD8 Positive Isolation Kit. RNA was extracted using the Ultrapure RNA Kit (Cwbiotech, Beijing, China). For quantitating RNA samples, a Qubit RNA HS Assay Kit (Thermo Fisher Scientific, PLeasanton CA, USA) was used. The Agilent RNA 6000 Nano Kit and Agilent 2100 Bioanalyzer were used for RNA integrity assessment. For each sample, $20 \mathrm{ng}$ of total RNA was reverse transcribed using SuperScript IV VILO Master Mix (Thermo Fisher Scientific), and then $25 \mathrm{ng}$ cDNA was target amplified using the Oncomine TCR Beta-LR Assay Kit (Thermo Fisher Scientific).

Libraries were purified and diluted 1:100 and quantified using the lon Library Quantitation Kit (Thermo Fisher Scientific) and then diluted to $25 \mathrm{pM}$ with low TE buffer. Samples were pooled on lon 530 chip for sequencing via the lon S5 System, followed by analysis via the lon Reporter. Total RNA of the Jurkat cell line is obtained from a clonotype with a T cell phenotype. T cell leukemia (Jurkat) total RNA is derived from a cell line consisting of a single T cell clonotype. For the control, running the Oncomine ${ }^{\mathrm{TM}}$ TCR Beta Assay on Jurkat Total RNA detected a single clonotype.

Equal numbers of available cells in CD8 ${ }^{+}$PD- $1^{+}\left(2 \sim 5 \times 10^{4}\right.$ cells $)$ and $C D 8^{+}$PD- $1^{-}\left(2 \sim 5 \times 10^{4}\right.$ cells $) \mathrm{T}$ cell populations from each sample were collected for RNA extraction and subsequent TCR $\beta$ sequencing.

\section{Data processing and analysis}

Repertoire quality metrics analysis was performed by the Oncomine ${ }^{\mathrm{TM}}$ TCR Beta-LR Assay workflow in the Ion Reporter ${ }^{\text {TM }}$ Software. The Shannon index and Gini index were used to characterize the frequency and sequence features of clonotypes. The Gini index was calculated as $\frac{A}{A+B}$, in which, for a set of clone frequencies, A represents the difference between the total area under the line of equality and the area 
under the Lorenz curve, and B represents the area under the Lorenz curve. The index ranges from 0 to 1. Higher values indicate dissimilar clone sizes, while lower values indicate more similar clone sizes.

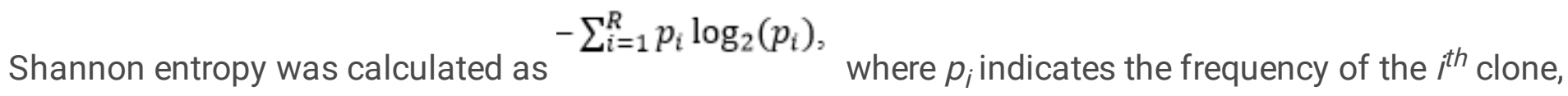
and $\mathrm{R}$ indicates the total number of clones[24]. Samples having many clones of similar frequencies will have high Shannon diversity. Subsequent analysis of the T cell receptor repertoire was performed using VDJtools [25], tcRpackages [26], Treemap [27], mothur [28].

The Mann-Whitney U-test was used to determine whether there were differences between the two groups. Analysis of covariance was initially used for the multiple group comparison. Correction for multiple tests was performed using false discovery rate method. The Wilcoxon signed-rank test was used for matched paired comparisons.

\section{Results}

\section{Extensive clonally expanded $\mathrm{CD} 8^{+} \mathrm{T}$ cell populations in the bone marrow of AML patients}

CDR3, a hypervariable region that consists of $a$ and $\beta$ chains and interacts with the MHC-peptide antigen complex, accounts for most of the antigen-reactive T cell diversity. We performed deep sequencing to assess the TCR $\beta$ CDR3 diversity from bone marrow (BM) and peripheral blood (PB) of AML patients and healthy donors. The distribution plot of the top 100 TCR clonotypes from bone marrow and peripheral blood of one AML patient and one healthy donor is shown in Fig. 1A. The graph demonstrates increased clonal expansion in the bone marrow of the AML patient compared to the other groups. Next, we sought to assess the extent of TCR repertoire diversity and clonal expansion by analyzing the total/unique clonotype ratio and highly expanded clones (HECs, clonotypes with a frequency of more than $0.1 \%$ in each sample[29]) ratio for all samples. Figure 1B shows that the total/unique clonotype ratios were higher in the bone marrow of AML patients than in the peripheral blood of AML patients and in the bone marrow and peripheral blood of healthy donors. A markedly higher frequency of highly expanded clones (HECs) was noted in the bone marrow and peripheral blood of AML patients than in those of healthy donors (Fig. 1C). In addition, the Shannon index and Gini index were used to evaluate the TCR repertoire diversity. Samples with expanded T cell clones of similar frequencies were found to be positively correlated with Shannon diversity index values. A lower value of the Gini index corresponded to a more uniform distribution of clone sizes. As shown in Fig. 2D, the Shannon index in bone marrow of $A M L$ patients was significantly higher than in peripheral blood of AML patients and bone marrow and peripheral blood of healthy donors; in contrast, the Gini index in the bone marrow of AML patients had a pronounced reduction compared with the other groups (Fig. 1E). Collectively, these findings showed that in $\mathrm{CD}^{+} \mathrm{T}$ cells from the bone marrow of AML patients, a decline in T cell repertoire diversity is closely associated with clonotypic expansion.

Comparison of overall usage of TCR $\beta$ V-J rearrangements in AML patients and healthy donors 
The CDR3 region combines the junction of the $V, D$, and $J$ gene segments and mainly accounts for the massive diversity of T cells. For several decades, it has been recognized that TCR diversity relies mainly on the hypervariable CDR3 region, and rearranged segments with the addition/subtraction of extra nucleotides at the recombination junctions contribute to $T$ cell diversity and antigen binding. Thus, the overall usage profiles of $\mathrm{V}, \mathrm{D}$, and $\mathrm{J}$ gene segments were analyzed. We identified a total of 60 distinguishable gene transcription segments from the TCR $\beta$ V (TRBV) loci, 2 from the TRBD loci, 13 from the TRBJ loci, and 780 rearrangements in the TRBV-J region. First, the usage profiles of TRBV-J rearrangements present in samples from different groups were compared. As shown in the graph in Fig. 2A, each rearrangement event in TRBV-J is denoted by a dot, with its size indicating the average frequency of the rearrangement in the sample group. Similar overall usage profiles of the rearranged TRBV/J segments were noted between bone marrow and peripheral blood from healthy donors (81 differentially expressed rearrangements) and between peripheral blood from AML patients and peripheral blood from healthy donors (62 differentially expressed rearrangements); however, differences were greater between bone marrow and peripheral blood from AML patients (176 differentially expressed rearrangements) and between bone marrow of AML patients and bone marrow of healthy donors (202 differentially expressed rearrangements) (Fig. 2B-E). Similarly, we found comparable usage patterns of TRBV gene segments between PB of healthy donors and BM of healthy donors and between PB of healthy donors and $\mathrm{PB}$ of $\mathrm{AML}$ patients but found relatively different usage patterns between $B M$ of healthy donors and BM of AML patients and between PB of AML patients and BM of AML patients (Figure S1). These data suggest that $\mathrm{CD} 8^{+} \mathrm{T}$ cells in the bone marrow of AML patients have specific expression of TRBV-J rearrangements, indicating that they may recognize bone marrow specific antigens.

\section{Comparison of identical clonotypes in AML patients and healthy donors}

We analyzed the CDR3 amino acid sequences of $C D 8^{+} T$ cells in peripheral blood and in bone marrow of 31 AML patients and 10 healthy donors to identify the presence of shared clones (i.e., shared CDR3 sequences) in the top 1,000 or 5,000 clones between any two sample pairs (Fig. 3A). Through intragroup analysis of the CDR3 sequences between different sample pairs, we found a higher ratio of identical T cell clones between sample pairs in the bone marrow of the AML patients (AML BM VS BM, $n=465$ ) when compared to sample pairs in other groups (AML PB VS PB, $n=465$ or healthy donor BM VS BM / PB VS PB, $n=45)(F i g .3 B)$. Intergroup analysis results indicated that there was no difference in the percent of identical T cell clones between sample pairs from different groups (AML patient BM vs. $P B, n=930$; healthy donor $\mathrm{BM}$ vs. $\mathrm{PB}, \mathrm{n}=90$; $\mathrm{AML}$ patient $\mathrm{BM}$ or $\mathrm{PB}$ vs. healthy donor $\mathrm{BM}$ or $\mathrm{PB}, \mathrm{n}=310$ ) (Fig. $3 \mathrm{C}$ ). $\mathrm{By}$ analyzing identical $T$ cell clones in the peripheral blood and bone marrow of AML patients or healthy donors (i.e., comparison between BM and PB of the same individual, $n=31$ or $n=10$ ), we found that the percent of identical $T$ cell clones between peripheral blood and bone marrow of the same individual was significantly higher than that between peripheral blood and bone marrow among different individuals. Moreover, the percentage of identical T cell clones between peripheral blood and paired bone marrow of the healthy donors was significantly higher than that in peripheral blood and paired bone marrow of the 
AML patients (Fig. 3D). These results suggested that clonal expansion of bone marrow $\mathrm{CD}^{+}{ }^{+}$cells in AML patients was highly specific.

\section{TCR $\beta$ repertoire variety and stability among CR patients and relapsed patients}

Thirty-one newly diagnosed patients were followed up after chemotherapy. In 12 patients who relapsed and 19 patients who had CR after chemotherapy, samples were collected for subsequent studies. Figure $4 \mathrm{~A}$ shows bone marrow $\mathrm{CD} 8^{+} \mathrm{T}$ cell clonal expansion in one relapsed patient and one sustained $\mathrm{CR}$ patient at new diagnosis and after chemotherapy. Each square represents a clone. A larger square size indicates greater clone, and different colors of the squares indicate different $\mathrm{V}$ genes. After discontinuation of chemotherapy, markedly expanded T cell clones were noted in the bone marrow from the relapsed patient but not from the patient who achieved CR. Looking more deeply into the clonal diversity of the TCR $\beta$ repertoires, we found considerably less diverse in relapse post chemotherapy group compared to the other groups (Fig. 4B). We further calculated the $\mathrm{V}$ gene usage of $\mathrm{CD} 8^{+} \mathrm{T}$ cells in this relapse patient, and the results showed that compared to the new diagnosis, the preferential usage pattern of some $V$ gene segments changed dramatically after relapse, and the number of different clones from the same $V$ gene usage was also remarkably different (Figure S2). To better investigate the CD8 ${ }^{+} \mathrm{T}$ cell clone characteristics, we analyzed the Shannon and Gini indexes of bone marrow T cells in all 31 patients at new diagnosis and after chemotherapy. Compared to the new diagnosis, the Shannon entropy of the relapsed patients had a marked reduction, whereas the Gini index increased significantly. No difference was observed in the Shannon and Gini indexes in patients with complete remission (Fig. 4C-D). By assessing the ratio of identical clones in the top 1,000 $\mathrm{T}$ clones in the bone marrow of the patients with complete remission or in relapsed patients at new diagnosis and after treatment, we found that the ratio of identical $\mathrm{T}$ clones in the relapsed patients before and after treatment was significantly lower than that in the complete remission group. However, when we increased the number of clones assessed to 5,000 , no difference was observed between the relapsed group and the complete remission group in terms of the ratio of identical T clones at new diagnosis and after chemotherapy (Fig. 4E). These results showed that compared to the AML patients who were in complete remission after chemotherapy, some of the $\mathrm{CD} 8^{+} \mathrm{T}$ cells of the patients who relapsed after treatment had massive clonal expansion and reduced clonal diversity and significant change in the T cell composition in the top 1,000 clones.

\section{TCR repertoire distribution of $\mathrm{CD} 8{ }^{+} \mathrm{T}$ cells based on $\mathrm{PD}-1$ expression}

As a dominant inhibitory receptor, $\mathrm{PD}-1$ is the pivotal mediator that induces $\mathrm{CD} 8^{+} \mathrm{T}$ cell exhaustion and contributes to T cell impairment. Several previous reports have suggested the functional involvement of PD-1 in AML progression [2, 18, 30, 31]. Compared to the time of diagnosis, for PD-1-expressing CD8 T cells, a significantly increased tendency in the bone marrow of relapsed patients and a decreased trend in the bone marrow of patients who had CR were noted in our study (Fig. 5A). To determine whether CD $8^{+} \mathrm{PD}-1^{+}$cells exhibited more clonal expansion, TCR $\beta$ deep sequencing of $C D 8^{+} \mathrm{PD}-1^{+}$and $\mathrm{CD} 8^{+} \mathrm{PD}-1^{-}$ T cells was performed. Figure 5B-E depicts the TCR repertoire distribution in 4 samples from one patient who relapsed and one patient who achieved sustained remission at new diagnosis and after 
chemotherapy. In all the samples analyzed, $\mathrm{CD} 8^{+} \mathrm{PD}-1^{+} \mathrm{T}$ cells were found to be more oligoclonal than CD8 ${ }^{+}$PD- $1^{-}$T cells. The top 50 clonotypes cumulative frequency made up $42.9 \%, 35.9 \%, 41.5 \%$, and $50 \%$ of the PD- $1^{+} \mathrm{T}$ cells total frequency at the time of $\mathrm{CR}$ diagnosis, $\mathrm{CR}$ post chemotherapy, relapse diagnosis, and relapse post chemotherapy, respectively, but only $18.2 \%, 15.8 \%, 20.4 \%$, and $29.6 \%$ of the PD- $1^{-}$total frequency at the time of CR diagnosis, CR post chemotherapy, relapse diagnosis, and relapse post chemotherapy, respectively (Fig. 5B and D). Since the abundance of CDR3 based on TCR sequencing of bulk CD $8+T$ cells can better reflect $T$ cell clonal expansion, we analyzed the distribution of the top 50 clonotypes in bulk CD8 ${ }^{+} \mathrm{T}$ cells in previous results of these two patients in $\mathrm{CD} 8{ }^{+} \mathrm{PD}-1^{+}$cells and $\mathrm{CD} 8{ }^{+}$ PD-1- $T$ cells. At both diagnosis and post chemotherapy, the clones with higher frequency and the overall frequency of the top 50 clonotypes in the $\mathrm{CD} 8^{+} \mathrm{PD}-1^{+}$group were higher than those in the $\mathrm{CD} 8^{+} \mathrm{PD}-1^{-}$ counterpart (Fig. 5C and E, Table S2-S5). Moreover, the top 50 prevalent clonotypes in the CD $8^{+}$PD- $1^{+}$ group were far less frequent in the PD- $1^{-}$counterpart (Figure S3). Analysis of all samples from the 5 relapsed patients and $19 \mathrm{CR}$ patients also revealed that the top 50 clonotypes in each $\mathrm{CD}^{+}$population were more frequently distributed in the $\mathrm{CD} 8^{+} \mathrm{PD}-1^{+}$group than in the $\mathrm{CD} 8^{+} \mathrm{PD}-1^{-}$group (Fig. $5 \mathrm{~F}$ ). Regarding the Shannon index, there were significant reductions in the $\mathrm{CD} 8^{+} \mathrm{PD}-1^{+}$group compared to the CD $8^{+}$PD- $1^{-}$group, indicating lower TCR repertoire diversity in the CD8 ${ }^{+}$PD- $1^{+}$group (Fig. 5G).

\section{Discussion}

$\mathrm{CD}^{+} \mathrm{T}$ cells are cytotoxic effector cells of the immune system that are involved in cell-mediated immunity. Cytotoxic T lymphocyte (CTL) reactivity depends mainly on the hypervariable TCR heterodimer CDR3, which is composed of TCRa and TCR $\beta$ chains and is responsible for recognition of cell surface MHC-peptide complexes [32]. Currently, our understanding of TCR repertoires in CD8 ${ }^{+} \mathrm{T}$ cells and the effect of chemotherapy on TCR repertoires in AML is still lacking. We performed a comprehensive analysis of TCR repertoires in $\mathrm{PB}$ and $\mathrm{BM} C D 8^{+} \mathrm{T}$ cells from 10 healthy donors and $31 \mathrm{AML}$ patients at diagnosis and post chemotherapy. The results reveal (i) diminished TCR repertoire diversity and increased clonal expansion in AML BM but not in PB; (ii) a higher identical clone ratio in AML BM; (iii) higher T cell clonal expansion post chemotherapy in relapsed patients compared to new diagnosis; and (iv) more expansion and lower diversity of TCR $\beta$ clonotypes in CD8 $8^{+} \mathrm{PD}-1^{+} \mathrm{T}$ cells than in $\mathrm{CD} 8^{+} \mathrm{PD}-1^{-} \mathrm{T}$ cells.

In the present study, we observed greater clonal expansion of $\mathrm{CD} 8^{+} \mathrm{T}$ cells in bone marrow but not in peripheral blood of AML patients when compared to healthy donors. Jian Zhang and colleagues reported higher clonal expansion in both T cells and B cells in peripheral blood of AML patients [15]. In contrast to their findings, we did not find clonal expansion of $C D 8^{+} \mathrm{T}$ cells in the peripheral blood of AML patients. This may be due to the different methods used for attaining TCR sequences and the heterogeneity of the samples. We obtained approximately 3,280,000 unique CD ${ }^{+}$TCR $\beta$ CDR3 sequences from 82 samples by using the target amplification method; in comparison, Jian Zhang and colleagues obtained 225,000 CDR3 sequences in TCR $a, \beta, \gamma$, and $\delta$ chains and 1,210,000 CDR3 sequences in B cell immunoglobulin (Ig) heavy and light chains from unselected bulk tumor RNA-seq data of 369 samples. Based on the data 
characteristics, we were able to better assess $\mathrm{CD} 8^{+} \mathrm{T}$ cell clones, whereas Jian Zhang et al. could analyze the overall $\mathrm{T}$ cell $\left(\mathrm{CD} 4^{+} \mathrm{T}\right.$ cells and $\mathrm{CD} 8^{+} \mathrm{T}$ cells) clone characteristics based on a larger sample size. At the same time, we found that compared with healthy donors, AML patients had a lower rate of identical $\mathrm{CD}^{+} \mathrm{T}$ cell clones between bone marrow and their own peripheral blood. In addition, compared to the time of diagnosis, the TOP $1000 \mathrm{CD} 8^{+} \mathrm{T}$ cell clones in the bone marrow of patients who relapsed after chemotherapy showed greater changes. Originating from myeloid hematopoietic progenitors, AML cells can grow and differentiate within the bone marrow microenvironment [33]. Additionally, bone marrow has been proven to be a preferential site for T cells specific for blood-borne or non-small-cell lung cancer associated antigens $[34,35]$. These features may support our notion that $C D 8^{+} T$ cell clones in the bone marrow of AML patients have special characteristics.

Several studies reported increased levels of PD-1 as well as multiple co-expressing inhibitor receptors (IRs) on $\mathrm{CD}^{+} \mathrm{T}$ cells of AML patients at diagnosis $[18,19]$. Longitudinal observation of changes in the IRs expression pattern indicate that PD-1 and Tim3, together with several other coinhibitory receptors, are increased in bone marrow $\mathrm{CD} 8^{+} \mathrm{T}$ cells in non-responders and reduced in responders post chemotherapy $[2,36]$. Our analysis of TCR $\beta$ sequences showed that TCR $\beta$ clonotypes in $C D 8^{+} P D-1^{+}$populations were more oligoclonal than those in $\mathrm{CD} 8^{+} \mathrm{PD}-1^{-} \mathrm{T}$ populations. These observations highlight the importance of further evaluating TCR clonotypic frequency and unique phenotypic traits of $C D 8^{+} T$ cells in $A M L$ to identify patient-specific repertoires of tumor-reactive $\mathrm{CD} 8^{+}$lymphocytes. For therapies that require isolation of T cells expressing inhibitory receptors [37,38], T cell exhaustion and dysfunction pose a major challenge. However, the present study and other recently published works suggested that CD8 ${ }^{+} \mathrm{PD}-$ $1^{+}$cells had extensive clonal expansion [21], produced IFN- $\gamma$ and lysed tumors in vitro [18]. This adds to evidence that the process of $\mathrm{T}$ cell dysfunction associated with co-expressing inhibitory receptors is reversible, and the enrichment of tumor-reactive cells for patient treatment is reproducible.

Our study has several limitations. First, higher disease heterogeneity and small sample size may have limited our ability to uncover special CDR3 amino sequences as biomarkers for AML characteristics or the exact baseline of TCR repertories diversity in association with prognosis. Second, isolation of the patientspecific repertoire of tumor-reactive $\mathrm{CD}^{+} \mathrm{T}$ cells was not performed, so the anti-leukemia effect was not evaluated. AML is characterized by reduced immunogenicity and has one of the lowest mutation rates compared to other cancer types [39]; nevertheless, it is the quality of mutations, not the mutational burdens, that may be of more significance in enhancing potent immune functions. Several other studies have shown that NPM1 mutation related TCRs correlates with enhanced anticancer effects and improved clinical outcomes [40,41]. Thus, isolation and recognition of antigens derived from common AML mutations such as NPM1, DNMT3A, and FLT3, identification of T cell receptors that can recognize these antigens, and analysis of related $T$ cell receptors in patients may be the logical starting point for the implementation of immunotherapy in AML. Third, owing to the scant sample availability, comprehensive comparison of patients between new diagnosis and after chemotherapy was restricted. To extensively 
analyze the TCR repertoire discrepancies of relapsed patients before and after chemotherapy would provide valuable information, so further studies should be carried out in this field.

\section{Conclusions}

Our findings provide the first objective data for characterizing signatures of $\mathrm{CD} 8^{+} \mathrm{T}$ cell receptor repertoires at diagnosis and after chemotherapy in patients with AML. Sequence analysis of CDR3 has shown that $C D 8^{+} T$ cells in the bone marrow of AML patients display specific TCR $\beta$ clonal expansion, and higher expansion is found in patients who relapse after chemotherapy and PD- $1^{+} \mathrm{T}$ cells. The antitumor reactivity of individual TCRs can be harnessed in the future to develop tailored T cell therapies for AML patients, and understanding immune responses to chemotherapy is thus crucial for the development and use of immunotherapies to treat patients with AML.

\section{Abbreviations}

AML: acute myeloid leukemia; BM: bone marrow; CDR3: complementarity determining region 3; CR: Complete response; CTL: cytotoxic T lymphocyte; HECs: highly expanded clones; PB: peripheral blood; PD-1: programmed death-1; TCR: T cell receptor;

\section{Declarations}

\section{Acknowledgements}

We thank Drs. Wenqiang Zhang and Ye Feng for offering their insightful advice on this project.

\section{Authors' contributions}

ZXF and JSW: designed the study, performed experiments, analyzed data, and wrote the manuscript; QF, $\mathrm{XYK}$ and YC provided technical and material support; $\mathrm{XL}$ and DM collected the clinical data.

\section{Consent for publication}

The authors declare that they agree to submit the article for publication.

\section{Funding}

This study was supported by the National Natural Science Foundation of China (Grant No.81660616, Grant No.81960032) and Social Development Research Project of Guiczhou Province (Grant No. [2015]3036).

\section{Availability of data and materials}

The datasets used and analyzed during the current study are available from the corresponding author on a reasonable request. 


\section{Ethics approval and consent to participate}

The study was approved by the Institutional Review Board (IRB) of the Affiliated Hospital of Guizhou Medical University (16-03-10). Informed consent was obtained from all the healthy adult donors and AML patients enrolled in the study.

\section{Competing interests}

The authors declare no competing interests.

\section{References}

1. Ferrara, F. \& Schiffer, C. A. Acute myeloid leukaemia in adults. The Lancet. 2013; 381: 484-495.

2. Knaus, H. A., Berglund, S., Hackl, H., Blackford, A. L., Zeidner, J. F., Montiel-Esparza, R. et al. Signatures of $C D 8+T$ cell dysfunction in AML patients and their reversibility with response to chemotherapy. JCl insight. 2018; 3: e120974.

3. Khalil, D. N., Smith, E. L., Brentjens, R. J. \& Wolchok, J. D. The future of cancer treatment: immunomodulation, CARs and combination immunotherapy. Nature reviews. Clinical oncology. 2016; 13: 273-290.

4. Kenderian, S. S., Ruella, M., Shestova, O., Klichinsky, M., Aikawa, V., Morrissette, J. J. et al. CD33specific chimeric antigen receptor $T$ cells exhibit potent preclinical activity against human acute myeloid leukemia. Leukemia. 2015; 29: 1637-1647.

5. Casucci, M., Nicolis di Robilant, B., Falcone, L., Camisa, B., Norelli, M., Genovese, P. et al. CD44v6targeted $\mathrm{T}$ cells mediate potent antitumor effects against acute myeloid leukemia and multiple myeloma. Blood. 2013; 122: 3461-3472.

6. Daver, N., Garcia-Manero, G., Basu, S., Boddu, P. C., Alfayez, M., Cortes, J. E. et al. Efficacy, Safety, and Biomarkers of Response to Azacitidine and Nivolumab in Relapsed/Refractory Acute Myeloid Leukemia: A Nonrandomized, Open-Label, Phase II Study. Cancer discovery. 2019; 9: 370-383.

7. S. Gill, S. K. T., M. Ruella, O. Shestova, Y. Li, D. L. Porter, M. Carroll, G. Danet-Desnoyers, J. Scholler, S. A. Grupp, C. H. June, M. Kalos. Preclinical targeting of human acute myeloid leukemia and myeloablation using chimeric antigen receptor-modified T cells. Blood. 2014; 123, 2343-2354

8. Tawara, I., Kageyama, S., Miyahara, Y., Fujiwara, H., Nishida, T., Akatsuka, Y. et al. Safety and persistence of WT1-specific T-cell receptor gene-transduced lymphocytes in patients with AML and MDS. Blood. 2017; 130: 1985-1994.

9. Perna, F., Berman, S. H., Soni, R. K., Mansilla-Soto, J., Eyquem, J., Hamieh, M. et al. Integrating Proteomics and Transcriptomics for Systematic Combinatorial Chimeric Antigen Receptor Therapy of AML. Cancer Cell. 2017; 32: 506-519 e505.

10. Kim, M. Y., Yu, K. R., Kenderian, S. S., Ruella, M., Chen, S., Shin, T. H. et al. Genetic Inactivation of CD33 in Hematopoietic Stem Cells to Enable CAR T Cell Immunotherapy for Acute Myeloid Leukemia. Cell. 2018; 173: 1439-1453 e1419. 
11. Reiser, J. \& Banerjee, A. Effector, Memory, and Dysfunctional CD8(+) T Cell Fates in the Antitumor Immune Response. J Immunol Res. 2016; 2016: 8941260.

12. Cole, D. K., Miles, K. M., Madura, F., Holland, C. J., Schauenburg, A. J., Godkin, A. J. et al. T-cell receptor (TCR)-peptide specificity overrides affinity-enhancing TCR-major histocompatibility complex interactions. The Journal of biological chemistry. 2014; 289: 628-638.

13. Fozza, C., Barraqueddu, F., Corda, G., Contini, S., Virdis, P., Dore, F. et al. Study of the T-cell receptor repertoire by CDR3 spectratyping. Journal of immunological methods. 2017; 440: 1-11.

14. Jin, Z., Luo, Q., Lu, S., Wang, X., He, Z., Lai, J. et al. Oligoclonal expansion of TCR Vdelta T cells may be a potential immune biomarker for clinical outcome of acute myeloid leukemia. Journal of hematology \& oncology. 2016; 9: 126.

15. Zhang, J., Hu, X., Wang, J., Sahu, A. D., Cohen, D., Song, L. et al. Immune receptor repertoires in pediatric and adult acute myeloid leukemia. Genome medicine. 2019; 11: 73.

16. Morath, A. \& Schamel, W. W. alphabeta and gammadelta T cell receptors: Similar but different. Journal of leukocyte biology. 2020; 107: 1045-1055.

17. Sharpe, A. H. \& Pauken, K. E. The diverse functions of the PD1 inhibitory pathway. Nature reviews. Immunology. 2018; 18: 153-167.

18. Jia, B., Wang, L., Claxton, D. F., Ehmann, W. C., Rybka, W. B., Mineishi, S. et al. Bone marrow CD8 T cells express high frequency of PD-1 and exhibit reduced anti-leukemia response in newly diagnosed AML patients. Blood cancer journal. 2018; 8: 34.

19. Williams, P., Basu, S., Garcia-Manero, G., Hourigan, C. S., Oetjen, K. A., Cortes, J. E. et al. The distribution of T-cell subsets and the expression of immune checkpoint receptors and ligands in patients with newly diagnosed and relapsed acute myeloid leukemia. Cancer. 2018;

20. Laszlo, G. S., Gudgeon, C. J., Harrington, K. H. \& Walter, R. B. T-cell ligands modulate the cytolytic activity of the CD33/CD3 BiTE antibody construct, AMG 330. Blood cancer journal. 2015; 5: e340.

21. Gros, A., Robbins, P. F., Yao, X., Li, Y. F., Turcotte, S., Tran, E. et al. PD-1 identifies the patient-specific CD8(+) tumor-reactive repertoire infiltrating human tumors. The Journal of clinical investigation. 2014; 124: 2246-2259.

22. Han, J., Duan, J., Bai, H., Wang, Y., Wan, R., Wang, X. et al. TCR Repertoire Diversity of Peripheral PD$1(+)$ CD8(+) T Cells Predicts Clinical Outcomes after Immunotherapy in Patients with Non-Small Cell Lung Cancer. Cancer immunology research. 2020; 8: 146-154.

23. Glanville, J., Huang, H., Nau, A., Hatton, O., Wagar, L. E., Rubelt, F. et al. Identifying specificity groups in the T cell receptor repertoire. Nature. 2017; 547: 94-98.

24. Zhang, L., Cham, J., Paciorek, A., Trager, J., Sheikh, N. \& Fong, L. 3D: diversity, dynamics, differential testing - a proposed pipeline for analysis of next-generation sequencing $T$ cell repertoire data. BMC Bioinformatics. 2017; 18: 129.

25. Shugay, M., Bagaev, D. V., Turchaninova, M. A., Bolotin, D. A., Britanova, O. V., Putintseva, E. V. et al. VDJtools: Unifying Post-analysis of T Cell Receptor Repertoires. PLoS Comput Biol. 2015; 11: e1004503. 
26. Nazarov, V. I., Pogorelyy, M. V., Komech, E. A., Zvyagin, I. V., Bolotin, D. A., Shugay, M. et al. tcR: an R package for T cell receptor repertoire advanced data analysis. BMC Bioinformatics. 2015; $16: 175$.

27. Di Lorenzo, B., Ravens, S. \& Silva-Santos, B. High-throughput analysis of the human thymic Vdelta1(+) T cell receptor repertoire. Sci Data. 2019; 6: 115.

28. Schloss, P. D., Westcott, S. L., Ryabin, T., Hall, J. R., Hartmann, M., Hollister, E. B. et al. Introducing mothur: open-source, platform-independent, community-supported software for describing and comparing microbial communities. Appl Environ Microbiol. 2009; 75: 7537-7541.

29. Han, Y., Liu, X., Wang, Y., Wu, X., Guan, Y., Li, H. et al. Identification of characteristic TRB V usage in HBV-associated HCC by using differential expression profiling analysis. Oncoimmunology. 2015; 4: e1021537.

30. Zhou, Q., Munger, M. E., Highfill, S. L., Tolar, J., Weigel, B. J., Riddle, M. et al. Program death-1 signaling and regulatory $\mathrm{T}$ cells collaborate to resist the function of adoptively transferred cytotoxic T lymphocytes in advanced acute myeloid leukemia. Blood. 2010; 116: 2484-2493.

31. Kong, Y., Zhang, J., Claxton, D. F., Ehmann, W. C., Rybka, W. B., Zhu, L. et al. PD-1(hi)TIM-3(+) T cells associate with and predict leukemia relapse in AML patients post allogeneic stem cell transplantation. Blood cancer journal. 2015; 5: e330.

32. Chen, G., Yang, X., Ko, A., Sun, X., Gao, M., Zhang, Y. et al. Sequence and Structural Analyses Reveal Distinct and Highly Diverse Human CD8(+) TCR Repertoires to Immunodominant Viral Antigens. Cell reports. 2017; 19: 569-583.

33. Shafat, M. S., Gnaneswaran, B., Bowles, K. M. \& Rushworth, S. A. The bone marrow microenvironment - Home of the leukemic blasts. Blood reviews. 2017; 31: 277-286.

34. Feuerer, M., Beckhove, P., Garbi, N., Mahnke, Y., Limmer, A., Hommel, M. et al. Bone marrow as a priming site for T-cell responses to blood-borne antigen. Nature medicine. 2003; 9: 1151-1157.

35. Safi, S., Yamauchi, Y., Stamova, S., Rathinasamy, A., Op den Winkel, J., Junger, S. et al. Bone marrow expands the repertoire of functional $T$ cells targeting tumor-associated antigens in patients with resectable non-small-cell lung cancer. Oncoimmunology. 2019; 8: e1671762.

36. Dama, P., Tang, M., Fulton, N., Kline, J. \& Liu, H. Gal9/Tim-3 expression level is higher in AML patients who fail chemotherapy. Journal for immunotherapy of cancer. 2019; 7: 175.

37. Durgeau, A., Virk, Y., Corgnac, S. \& Mami-Chouaib, F. Recent Advances in Targeting CD8 T-Cell Immunity for More Effective Cancer Immunotherapy. Frontiers in immunology. 2018; 9: 14.

38. Farhood, B., Najafi, M. \& Mortezaee, K. CD8(+) cytotoxic T lymphocytes in cancer immunotherapy: A review. J Cell Physiol. 2019; 234: 8509-8521.

39. Cancer Genome Atlas Research, N., Ley, T. J., Miller, C., Ding, L., Raphael, B. J., Mungall, A. J. et al. Genomic and epigenomic landscapes of adult de novo acute myeloid leukemia. The New England journal of medicine. 2013; 368: 2059-2074.

40. Dyantha I. van der Lee, J.H. Frederik Falkenburg, Marieke Griffioen. Mutated nucleophosmin 1 as immunotherapy target in acute myeloid leukemia. The Journal of clinical investigation. 2019; 129(2):774-785: 
41. Greiner, J., Ono, Y., Hofmann, S., Schmitt, A., Mehring, E., Gotz, M. et al. Mutated regions of nucleophosmin 1 elicit both $\mathrm{CD} 4(+)$ and $\mathrm{CD} 8(+)$ T-cell responses in patients with acute myeloid leukemia. Blood. 2012; 120: 1282-1289.

\section{Figures}

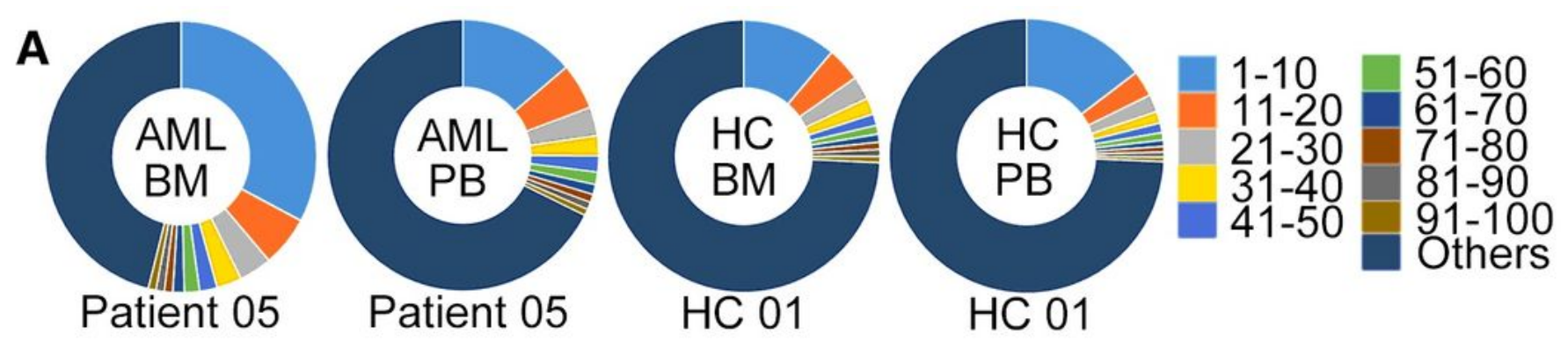

B

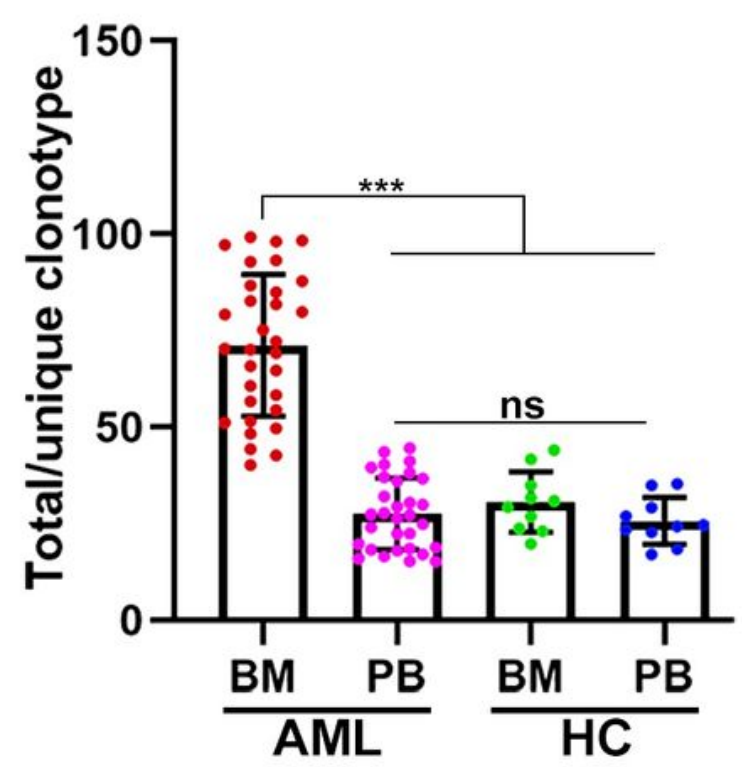

D

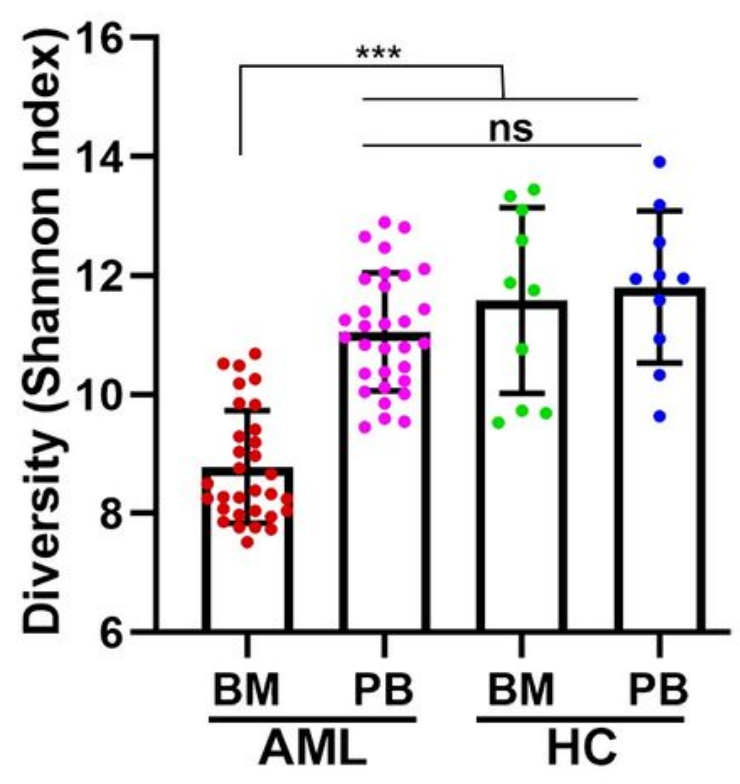

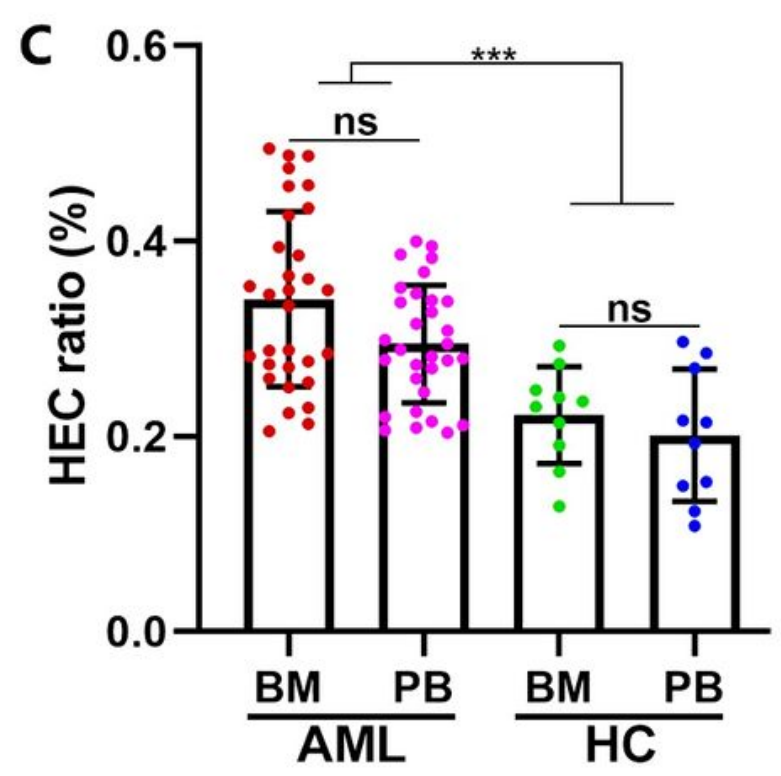

E

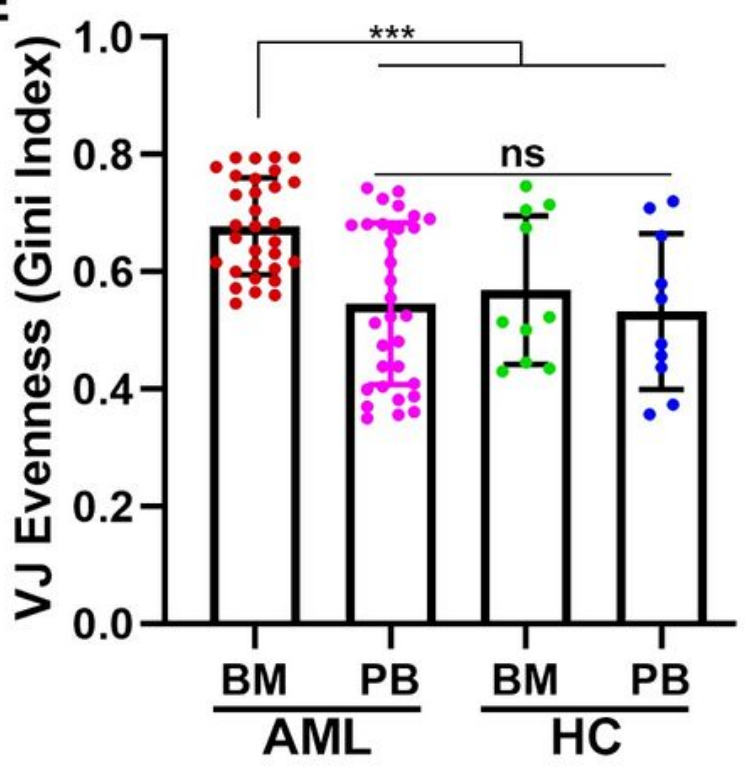

Figure 1 
Clonal expansion and diversity in PB and BM CD8+ T cells of AML patients and healthy donors (A) The distribution profile of the top 100 clonotypes from the BM and PB of one AML patient and one healthy donor as depicted in a pie chart diagram. The TCR repertoire diversity was evaluated by the total/unique clonotype ratio (B), the HEC ratio (C), the Shannon diversity index (D), the Gini index (E) in four study groups containing BM $(n=31)$ and PB $(n=31)$ samples obtained from AML patients and BM $(n=10)$ and PB $(n=10)$ samples obtained from healthy donors. $A$ dot is used to represent one patient or one donor sample. ns, indicates not significant; *** indicates $\mathrm{P}<0.01$.

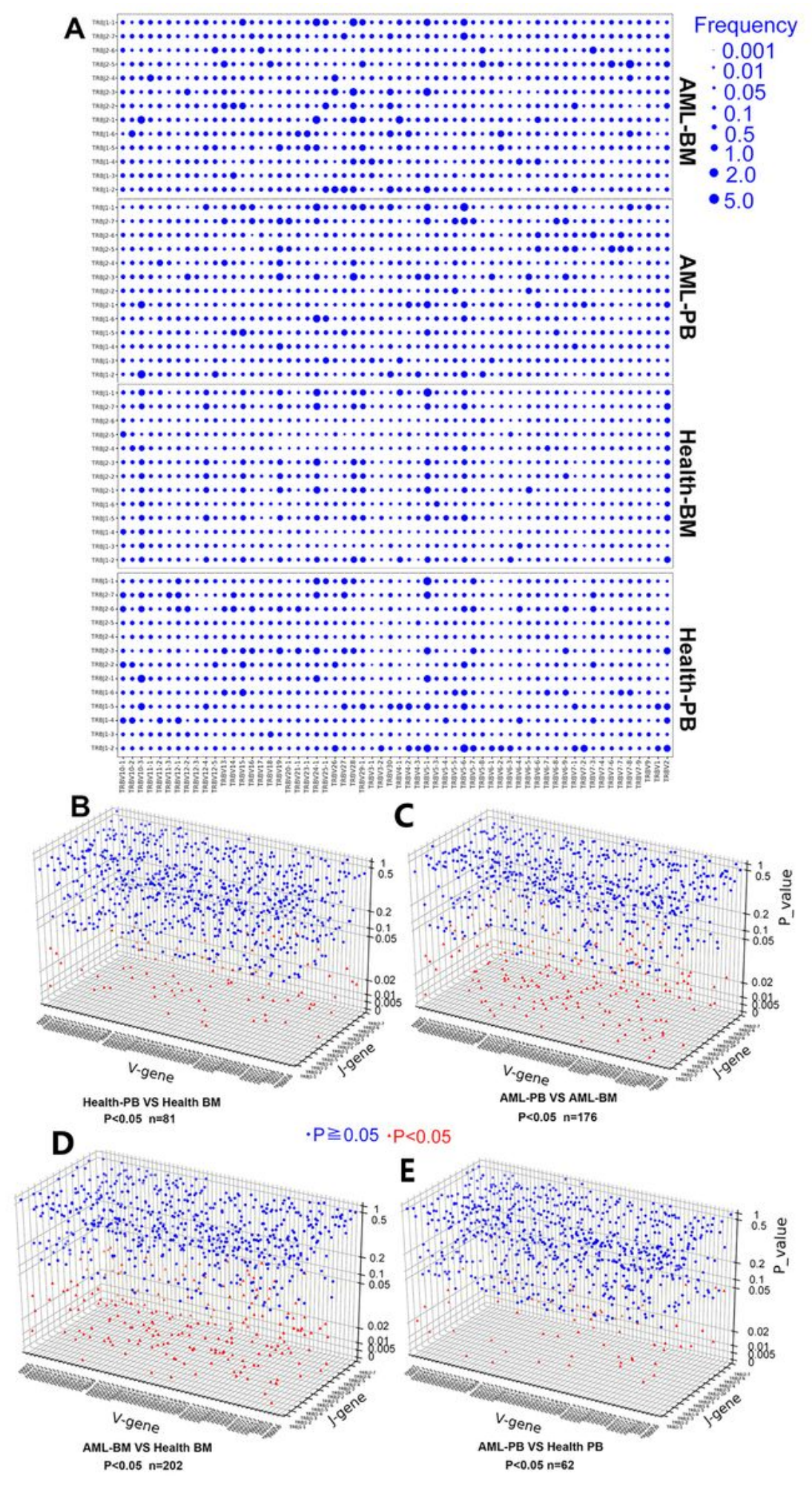


Figure 2

Usage patterns of TCR $\beta$ V-J rearrangements in the PB and BM CD8+ T cells of AML patients and healthy donors (A) Dot plots depicting the mean frequency distribution of TCR $\beta$ V-J gene rearrangements for $\mathrm{CD} 8+\mathrm{T}$ cells from the four groups. The variations in TCR $\beta$ V-J rearrangements between normal PB vs. healthy $B M(B), A M L P B$ vs. AML BM (C), AML BM vs. healthy BM (D), and AML PB vs. healthy PB (E) were compared. Blue circle, $\mathrm{P}<0.05$; red triangle, $\mathrm{P} \geq 0.05$.
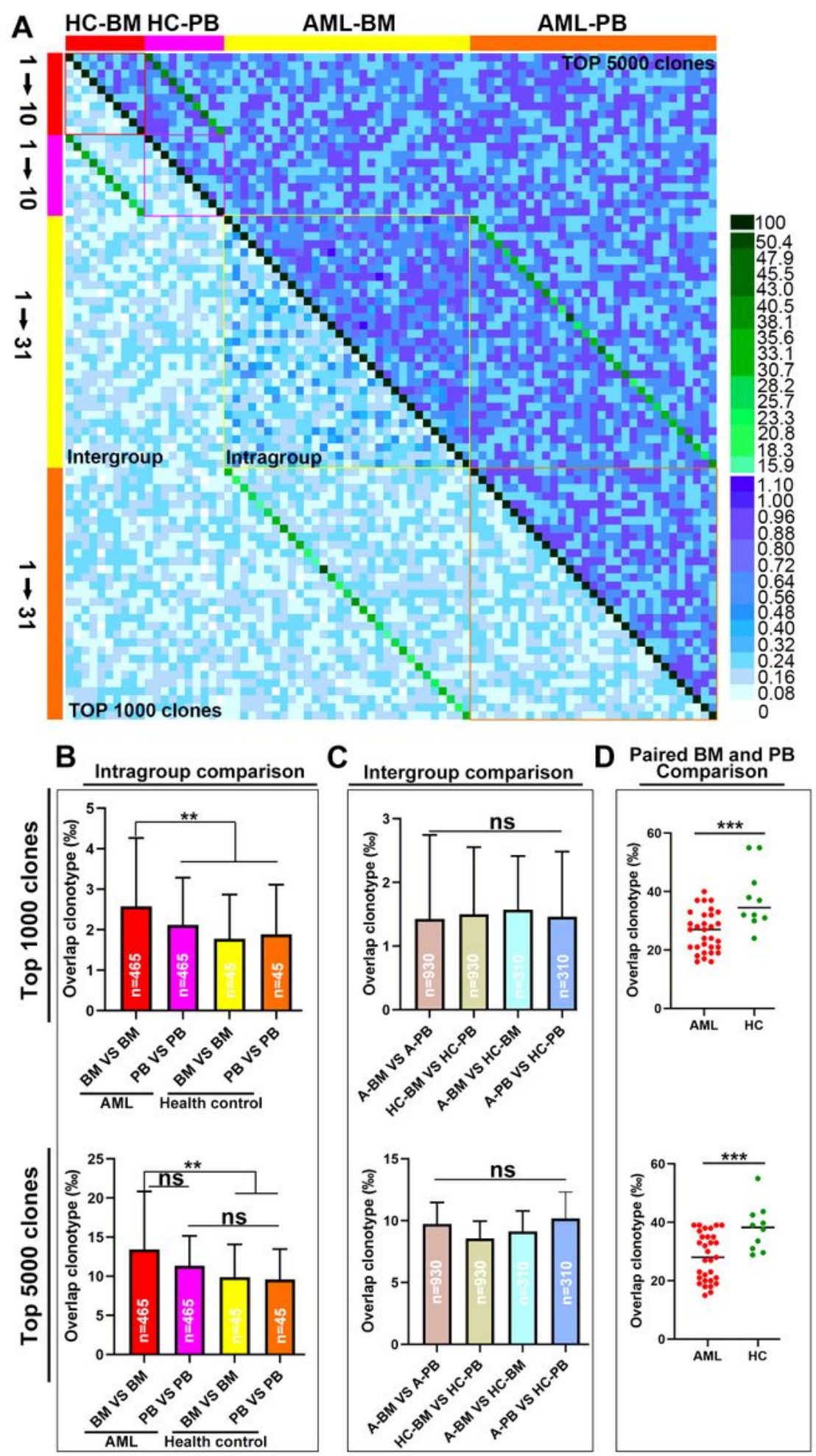


\section{Figure 3}

The metric of the TCR $\beta$ repertoire overlap in PB and BM CD8+ T cells of AML patients and healthy donors (A) A heat map was plotted to assess the similarity in the top 1000 most abundant CDR3 amino sequences or the top 5000 most abundant CDR3 amino sequences between any two samples. (B) Based on the top 1000 or top 5000 most abundant CDR3 amino sequences, repertoire overlap analysis was performed within each group of samples and (C) between groups of samples. (D) Based on the top 1000 or top 5000 most abundant CDR3 amino sequences, overlaps between BM and PB of the same individual in AML patients and healthy donors were compared. A dot is used to represent patient samples. ns, indicates not significant; $* * P<0.05 ; * \star * P<0.01$. 

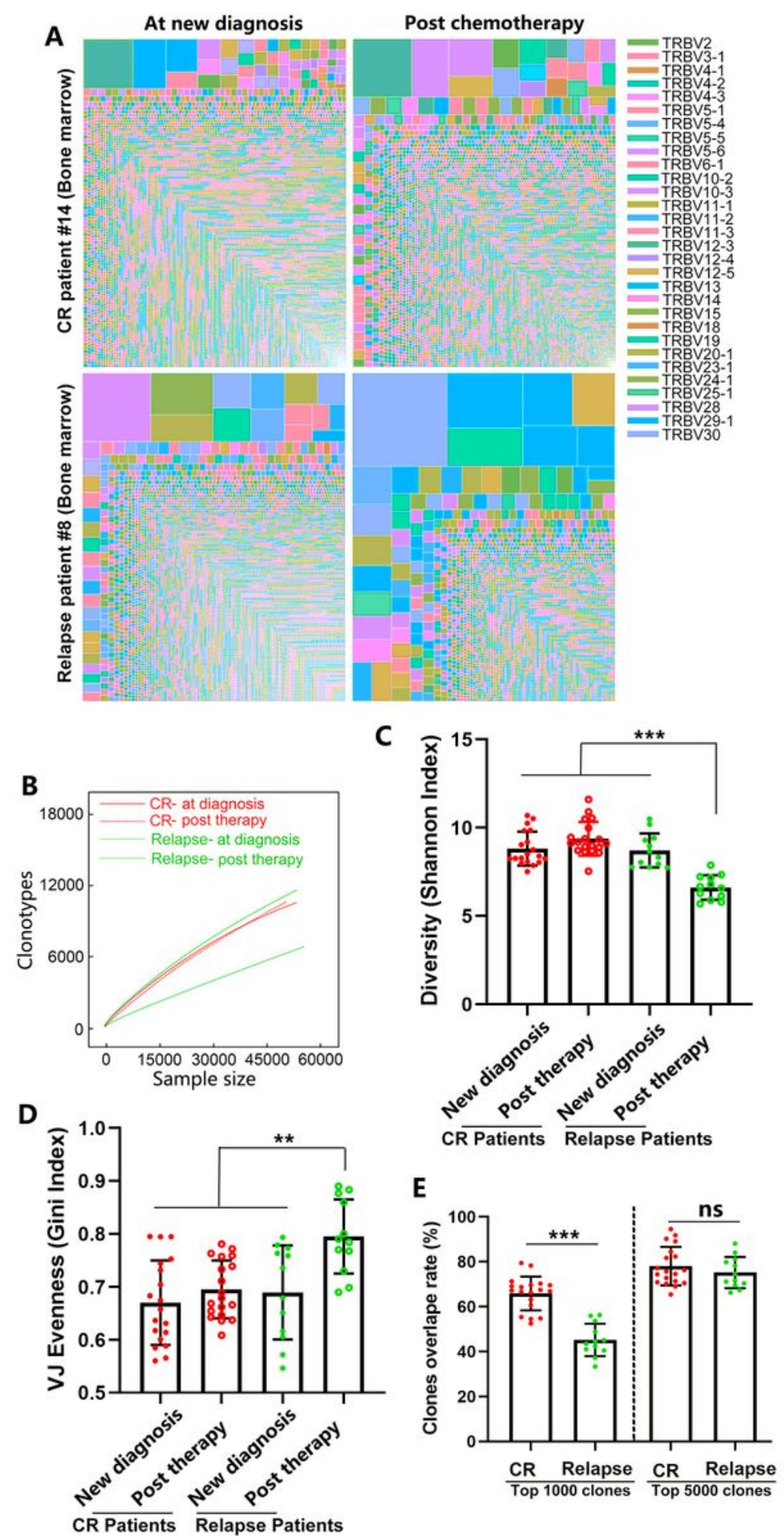

\section{Figure 4}

High degree of clonal expansion in BM CD8+ T cells of relapsed AML patients (A) Treemap showing the clone repertoires in one patient who relapsed and one patient who remained in remission at new diagnosis and post chemotherapy. Each amino acid sequence was perceived as a separate clonotype, which was represented by a square, and the frequency was indicated by its area; different $\mathrm{V}$ genes are represented by different colors. (B) Rarefaction analysis of repertoires from one patient who relapsed and 
one patient who remained in remission at new diagnosis and post chemotherapy. Number of unique clonotypes in a subsample is plotted against its size (number of TCR cDNA molecules). The diversities of the $T$ cell receptor repertoire in 12 patients who relapsed and 19 patients who had CR after chemotherapy were characterized by computing the Shannon index (C) and the Gini index (D). A dot is used to represent patient samples. (E) Repertoire overlaps were compared at new diagnosis and post chemotherapy in the BM of the same individual based on the top 1000 or top 5000 clonotypes. CR patients: patients who were newly diagnosed with AML and remained in complete remission after chemotherapy. Relapsed patients: patients who were newly diagnosed with AML and relapsed after chemotherapy. ns, indicates not significant; ${ }^{\star *} \mathrm{P}<0.01 ; * \star \star \mathrm{P}<0.001$. 

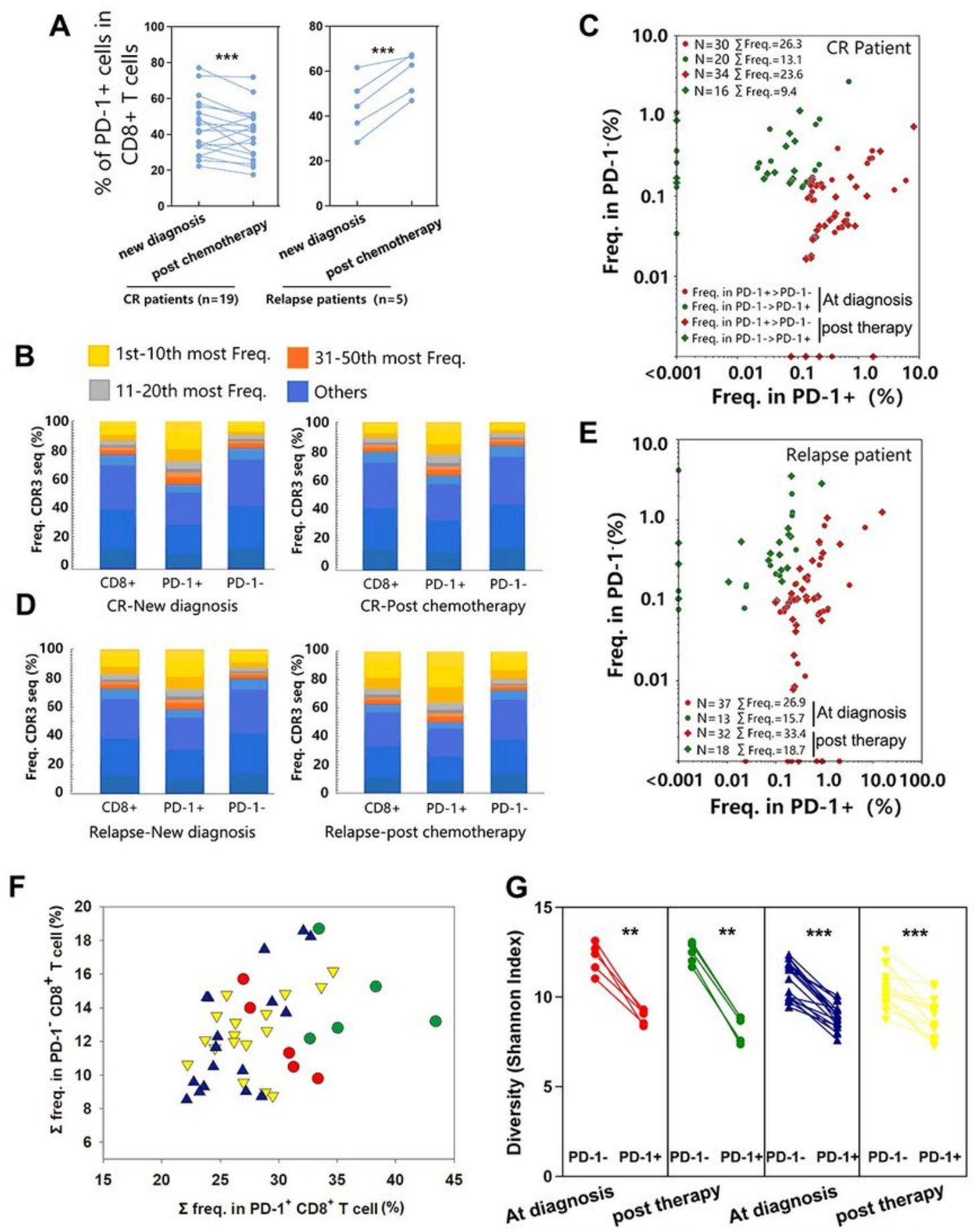

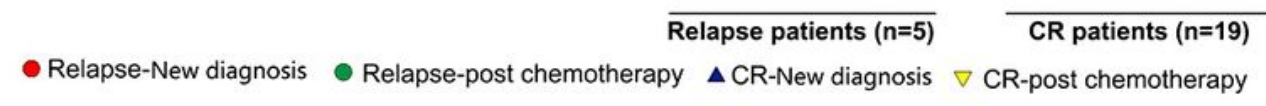

\section{Figure 5}

TCR repertoire distribution of CD8+ T cells based on PD-1 expression in CR and relapsed AML patients In 5 patients who relapsed and 17 patients who had CR after chemotherapy, samples were collected for subsequent studies. (A) Expression of PD-1 was determined by flow cytometry. (B-F) Clonotype distribution of CD8+ $T$ cells was associated with PD-1 expression. $C D 8+T$ cells were sorted from one patient who had CR (B and $C$ ) and one relapsed patient ( $D$ and $E$ ) based on the expression of PD-1. mRNA 
extraction and deep sequencing of TCR $\beta$ CDR3 were performed. (B and D) Analysis of TCR $\beta$ repertoire clonal frequency in CD8+ (as shown above), CD8+PD- $1+(4 \times 104$ cells), and CD8+PD-1- $(4 \times 104$ cells) T cells. The top 1 to 10,11 to 20 , and 21 to 50 clonotype abundances, as well as the remaining clonotypes, were shown. ( $C$ and $E$ ) The distribution of the top 50 frequent clonotypes in CD8+ T cells was compared between the CD8+ PD-1+ populations and CD8+ PD-1- populations. Each dot represents a clonotype from newly diagnosed samples; each rhombus represents a clonotype from samples post chemotherapy. Red represents the frequency of clonotypes in CD8+ PD-1+ > CD8+ PD-1-; green represents the frequency of clonotypes in CD8+ PD-1- > CD8+ PD-1+. The total frequency ( $\Sigma$ freq.) of the clonotypes in each of the populations is cumulative. (F) The top 50 clonotypes in CD8+ T cells were frequently distributed in CD8+ PD-1+ populations and CD8+ PD-1- populations. Red dots represent samples collected at new diagnosis from patients who relapsed post chemotherapy; green dots represent samples collected after treatment from patients who relapsed post chemotherapy; blue triangles represent samples collected at new diagnosis from patients with sustained CR post chemotherapy; yellow triangles represent samples collected after treatment from patients with sustained CR post chemotherapy; $(\mathrm{G})$ Shannon index comparison between CD8+ PD-1+ populations and CD8+ PD-1- populations. ${ }^{* * P}<0.05 ;{ }^{\star \star *} \mathrm{P}<0.01$.

\section{Supplementary Files}

This is a list of supplementary files associated with this preprint. Click to download.

- SupplementaryInformation.docx

- SupplementaryInformation.docx 